\title{
ТОПОГРАФО-АНАТОМИЧЕСКОЕ ОБОСНОВАНИЕ ВОЗМОЖНОСТИ ИСПОЛЬЗОВАНИЯ «SIЕА» ЛОСКУТА ПРИ РЕКОНСТРУКЦИИ МОЛОЧНОЙ ЖЕЛЕЗЫ
}

\author{
(C) Черных А.В., Закурдаев Е.И., Якушева Н.В., Закурдаева М.П.
}

\author{
Кафедра оперативной хирургии с топографической анатомией \\ Воронежского государственного медицинского университета им. Н.Н. Бурденко, Воронеж \\ E-mail: ezakurdaev@rambler.ru
}

\begin{abstract}
Исследовано 40 нефиксированных трупов лиц женского пола, умерших в возрасте 53,8 $\pm 4,4$ года. До аутопсии на каждом трупе измерялась длина паховой связки. На аутопсии трупов сначала определялось расположение поверхностной надчревной артерии и вены относительно уровня середины паховой связки. Затем измерялся диаметр поверхностной надчревной артерии и вены на уровне паховой связки. В исследованном секционном материале поверхностная надчревная артерия отмечена в 90\%, а вена - в 100\% наблюдений. Расстояние между поверхностной надчревной артерией и веной на уровне паховой связки составило 1,6 $\pm 0,5$ см. Диаметр поверхностной надчревной артерии на уровне паховой связки составил $1,7 \pm 0,2$ мм, а вены - 2,1 10,4 мм. Установлена корреляционная зависимость между длиной паховой связки и расстоянием между поверхностными надчревными сосудами на уровне паховой связки (r=0,75; $\mathrm{p}=0,01)$.
\end{abstract}

Ключевые слова: реконструкция молочной железы, аутопластика, передняя брюшная стенка, кровоснабжение.

\section{TOPOGRAPHIC-ANATOMICAL STUDY OF USING «SIEA» FLAP FOR BREAST RECONSTRUCTION \\ Chernykh A.V., Zakurdaev E.I., Yakusheva N.V., Zakurdaeva M.P. \\ Department of Operative Surgery with Topographic Anatomy \\ of N.N. Burdenko Voronezh State Medical University, Voronezh}

The features of constitutional and variant anatomy of the superficial epigastric vessels were studied. 40 female cadavers having died at the age of 53.8 \pm 4.4 years were examined. Before autopsy the length of inguinal ligament was measured on each corpse.During autopsy the location of superficial epigastric artery and vein was determined relative to the mid-level of inguinal ligament. Then the diameter of the superficial epigastric artery and vein was measured at the level of the inguinal ligament. In the investigated dissected material the superficial epigastric artery was observed in $90 \%$ and vein - in $100 \%$ of cases. The distance between the superficial epigastric artery and vein at the level of inguinal ligament was $1.6 \pm 0.5 \mathrm{~cm}$. The diameter of the superficial epigastric artery at the level of the inguinal ligament was $1.7 \pm 0.2 \mathrm{~mm}$ and vein $-2.1 \pm 0.4 \mathrm{~mm}$. The correlation between the length of inguinal ligament and the distance between the superficial epigastric vessels at the level of inguinal ligament $(\mathrm{r}=0.75 ; \mathrm{p}=0.01)$ was established.

Keywords: breast reconstruction, autologous reconstruction, abdominal wall, blood supply.

Наиболее распространенным онкологическим заболеванием у женщин является рак молочной железы [1-4]. Ежегодно заболевают около 40000 женщин и около 20000 умирают от этой болезни [5-13]. Важным методом лечения рака молочной железы является радикальная операция, которая заключается в удалении пораженной опухолью молочной железы.

Большой объем хирургического вмешательства по поводу рака молочной железы обусловливает необходимость в реконструкции данного органа. Очень часто пластику молочной железы выполняют с использованием силиконовых имплантантов, которые, однако, в 20\% быстро сморщиваются, что требует повторной операции $[9,11]$.

Реконструкцию молочной железы после мастэктомии достаточно часто выполняют с использованием аутопластических методик. Чаще всего в область дефекта пересаживают лоскут кожи, подкожной клетчатки и прямых мышц живота («TRAM» лоскуm) $[6,10,12]$. Данная методика со- провождается хорошими эстетическими результатами, но весьма травматична: у 2,6\% больных возникают послеоперационные грыжи [7, 12].

При аутопластической реконструкции молочной железы многие хирурги используют лоскут кожи и подкожной клетчатки передней брюшной стенки на питающей ножке из ветвей глубоких надчревных сосудов («DIEP» лоскуm) [8, 6, 10]. Данная методика менее травматична, но технические сложнее, так как требует выделения ветвей глубоких надчревных сосудов из толщи прямых мышц живота.

$\mathrm{B}$ настоящее время активно изучается «SIEA» пластика, когда для реконструкции молочной железы используется лоскут кожи и подкожной клетчатки передней брюшной стенки на питающей ножке из поверхностных надчревных сосудов $[8,10,11]$. Данная методика технически проще, но в 1,9-12,6\% осложняется некрозом и отторжением пересаженных тканей $[7,12]$. 


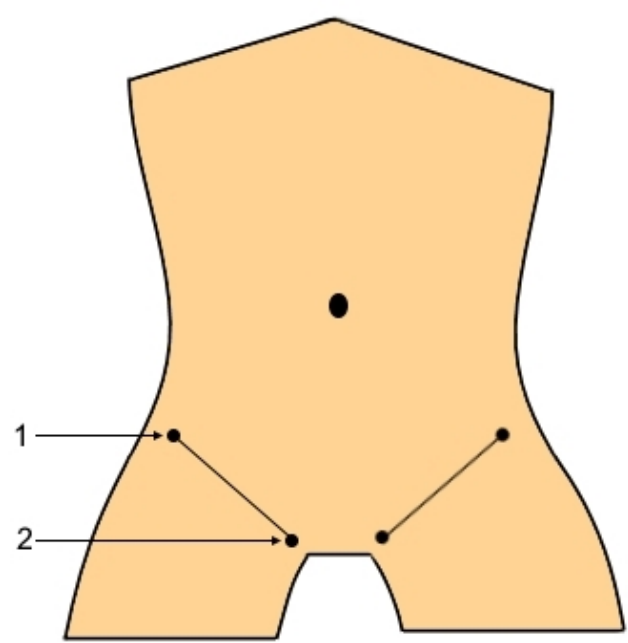

Рис. 1. Методология измерения длины паховой связки.

Обозначения: 1 - передняя верхняя ость подвздошной кости, 2 - лобковый бугорок.

При планировании и проведении реконструкции молочной железы с использованием «SIEA» лоскута важно учитывать наличие или отсутствие поверхностной надчревной артерии, взаимоотношение поверхностной надчревной артерии и одноименной вены, диаметр сосудов [5-13]. Однако указанные вопросы скудно освещены в доступной литературе.

В связи с вышеизложенным была поставлена цель исследования - изучить особенности типовой и вариантной анатомии поверхностных надчревных сосудов в прикладном аспекте.

\section{МАТЕРИАЛЫ И МЕТОДЫ ИССЛЕДОВАНИЯ}

Работа выполнена на 40 нефиксированных трупах лиц женского пола, умерших в возрасте $53,8 \pm 4,4$ года. Исследование было одобрено этическим комитетом при ФГБОУ ВО «Воронежский государственный медицинский университет им. Н.Н. Бурденко» Минздрава РФ.

До аутопсии на каждом трупе измерялась длина паховой связки - расстояние между передней верхней остью подвздошной кости и лобковым бугорком (рис. 1).

На аутопсии трупов сначала определялось расположение поверхностной надчревной артерии и вены относительно уровня середины паховой связки. Затем с использованием штангенциркуля измерялся диаметр поверхностной надчревной артерии и вены на уровне паховой связки.

При статистической обработке результатов исследования определялись среднее арифметическое (М) и стандартная ошибка среднего (m). Достоверность различий между количественными переменными определялась с использованием критерия Манна-Уитни. При оценке зависимости между переменными вычислялся коэффициент корреляции Спирмена. Различия считались значимыми при доверительной вероятности не менее $95 \%(\mathrm{p} \leq 0,05)$.

\section{РЕЗУЛЬТАТЫ ИССЛЕДОВАНИЯ И ИХ ОБСУЖДЕНИЕ}

Обязательным условием возможности использования «SIEA» лоскута в пластической хирургии является наличие поверхностной надчревной артерии и вены. По данным М. Fathi et al. [6], C.M. Reardon et al. [10], частота встречаемости поверхностной надчревной артерии достигает 100\%. В исследованиях E. Fukaya et al. [7] поверхностная надчревная артерия наблюдалась в $65 \%$.

Наши данные согласуются с результатами исследования J.C. Selber et al. [12]. Так, на изученном секционном материале поверхностная надчревная артерия с обеих сторон отмечена в 28 (70\%), а вена - во всех наблюдениях (рис. 2). В 4 (10\%) случаях поверхностная надчревная артерия отсутствовала с обеих сторон. В остальных 8 (20\%) наблюдениях поверхностная надчревная артерия отмечалась с одной стороны, чаще слева $(6(75 \%))$.

При выкраивании «SIEA» лоскута с целью реконструкции молочной железы после мастэктомии важно знать расположение поверхностных надчревных сосудов. Относительно середины паховой связки поверхностная надчревная артерия всегда располагалась латерально, а вена - медиально. Замечено, что справа поверхностная надчревная артерия находилась ближе к середине паховой связки, чем слева (табл. 1). 

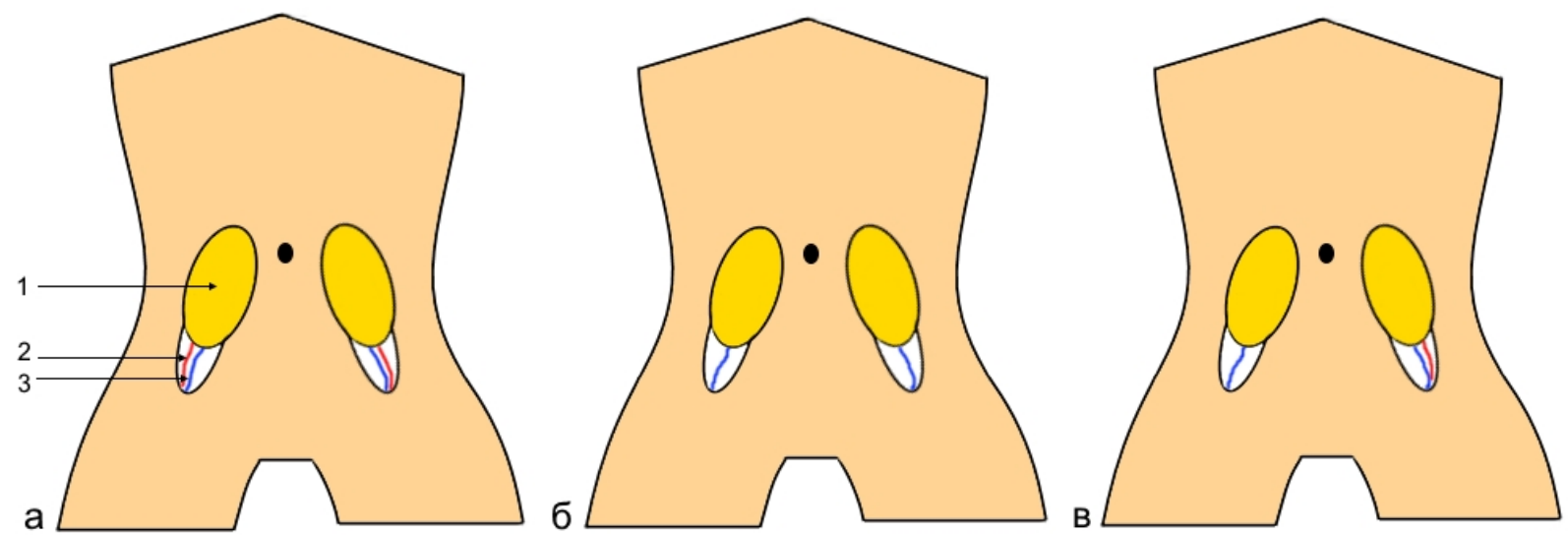

Рис. 2. Варианты топографии поверхностных надчревных сосудов: а - наличие артерии и вены с обеих сторон, б - отсутствие артерии с обеих стороны, в - отсутствие артерии с одной стороны.

Обозначения: 1 - выкроенный лоскут кожи и подкожной клетчатки из передней брюшной стенки («SIEA» лоскуm), 2 - поверхностная надчревная артерия, 3 - поверхностная надчревная вена.

Таблица 1

Расположение поверхностной надчревной артерии и вены относительно середины паховой связки, $\mathrm{M} \pm \mathrm{m}$

\begin{tabular}{|l|c|c|c|}
\hline \multirow{2}{*}{\multicolumn{1}{c|}{ Исследуемый кровеносный сосуд }} & \multicolumn{3}{|c|}{ Расстояние от между кровеносным сосудом } \\
\cline { 2 - 4 } & Справа & Слева & Среднее \\
\hline Поверхностная надчревная артерия & $0,6 \pm 0,1^{*}$ & $1,1 \pm 0,2$ & $0,8 \pm 0,2$ \\
\hline Поверхностная надчревная вена & $1,0 \pm 0,2$ & $0,8 \pm 0,3$ & $0,9 \pm 0,1$ \\
\hline
\end{tabular}

Примечание: * - различия между сторонами измерений статистически значимы при $\mathrm{p}=0,01$.

Таблица 2

Расстояние между поверхностными надчревными сосудами на уровне паховой связки, $\mathrm{M} \pm \mathrm{m}$

\begin{tabular}{|c|c|c|c|}
\hline Исследуемый параметр & Справа & Слева & Среднее \\
\hline Расстояние между поверхностными надчревными сосудами, см & $1,6 \pm 0,2^{*}$ & $1,9 \pm 0,1$ & $1,7 \pm 0,2$ \\
\hline
\end{tabular}

Примечание: * - различия между сторонами измерений статистически значимы при $\mathrm{p}=0,05$

Таблица 3

Диаметр поверхностной надчревной артерии и вены на уровне паховой связки, $\mathrm{M} \pm \mathrm{m}$

\begin{tabular}{|l|c|c|c|}
\hline \multirow{2}{*}{\multicolumn{2}{|c|}{ Исследуемый кровеносный сосуд }} & \multicolumn{3}{|c|}{ Диаметр кровеносного сосуда, мм } \\
\cline { 2 - 4 } & Справа & Слева & Среднее \\
\hline Поверхностная надчревная артерия & $1,8 \pm 0,1$ & $1,6 \pm 0,2$ & $1,7 \pm 0,2$ \\
\hline Поверхностная надчревная вена & $2,0 \pm 0,2$ & $2,3 \pm 0,5$ & $2,1 \pm 0,4$ \\
\hline
\end{tabular}

Примечание: статистически значимых различий между сторонами измерений не установлено - $>>0,05$.

Данные литературы свидетельствуют о том, что хорошее приживление «SIEA» лоскута достигается лишь в тех случаях, когда расстояние между сосудами составляет не более 1,8 см [5, 11]. По данным проведенного исследования, расстояние между поверхностной надчревной артерией и веной на уровне паховой связки составило $1,6 \pm 0,5$ см. Отмечено, что справа расстояние между поверхностными надчревными сосудами было достоверно больше (табл. 2).

При пересадке «SIEA» лоскута важно подключить его питающие сосуды к периферическим сосудами области пластики, что возможно при диаметре сосудов лоскута более
1,5 мм $[8,12,10]$. По данным M. Fathi et al. [6], диаметр поверхностной надчревной артерии

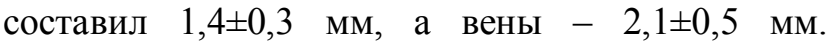
В работах C.M. Reardon et al. [10] указывается только диаметр поверхностной надчревной артерии - 1,9 мм. Согласно X. Wang et al. [12], диаметр поверхностной надчревной артерии составил 1,5 мм, а вены - 2,3 мм. Исследования B.J. Kim et al. [8] показали, что диаметр поверхностной надчревной артерии составил $1,2 \pm 0,4$ мм, а вены $-1,4 \pm 0,3$ мм.

Полученные в проведенном исследовании данные несколько расходятся с литературными. Так, на изученном секционном материале 
Таблица 4

Расстояние между поверхностными надчревными сосудами в зависимости от типа паховой связки, $\mathrm{M} \pm \mathrm{m}$

\begin{tabular}{|l|c|c|c|}
\hline \multirow{2}{*}{\multicolumn{2}{|c|}{ Тип паховой связки }} & \multicolumn{3}{|c|}{ Расстояние между поверхностными надчревными } \\
& \multicolumn{3}{|c|}{ сосудами, см } \\
\cline { 2 - 4 } & Справа & Слева & Среднее \\
\hline Протяженная (более 15 см) & $1,8 \pm 0,1^{*}$ & $2,1 \pm 0,2^{*}$ & $1,9 \pm 0,1^{*}$ \\
\hline Обычная (12-15 см) & $1,6 \pm 0,2$ & $1,9 \pm 0,1$ & $1,7 \pm 0,2$ \\
\hline Короткая (менее 12 см) & $1,3 \pm 0,1^{* *}$ & $1,6 \pm 0,1^{*}$ & $1,4 \pm 0,2^{* *}$ \\
\hline
\end{tabular}

Примечание: * - различия между типами паховой связки статистически значимы при $\mathrm{p}=0,05 ;$ ** - при $\mathrm{p}=0,01$.

диаметр поверхностной надчревной артерии на уровне паховой связки составил $1,7 \pm 0,2$ мм, а вены - 2,1 $\pm 0,4$ мм (табл. 3). Из этого следует, что диаметр исследованных кровеносных сосудов соответствует требованиям пластических хирургов по выполнению микрососудистых анастомозов.

На следующем этапе работы были изучены типовые особенности топографии поверхностных надчревных сосудов. С этой целью сначала исследована длина паховой связки - расстояние между передней верхней остью подвздошной кости и лобковым бугорком. Результаты показали, что длина паховой связки составила $13,5 \pm 2,6$ cм.

Затем с использованием рангового корреляционного анализа Спирмена установлена зависимость между длиной паховой связки и расстоянием между поверхностной надчревной артерией и веной на уровне паховой связки $(\mathrm{r}=0,75 ; \mathrm{p}=0,01)$. Полученные данные свидетельствуют о том, что с увеличением длины паховой связки увеличивается и расстояние между поверхностными надчревными сосудами.

Далее полученные данные были систематизированы, в результате установлены типы паховой связки: протяженная (длина более 15 см), обычная (12-15 см), короткая (менее 12 см). В исследованной выборке чаще всего наблюдался обычный тип паховой связки 24 (60\%). Протяженная паховая связка отмечена в 12 (30\%), а короткая - в 4 (10\%) наблюдениях.

После этого изучено расстояние между поверхностной надчревной артерией и веной в зависимости от типа паховой связки. Результаты исследования показали, что у лиц с протяженной паховой связкой наблюдались максимальные показатели расстояния между кровеносными сосудами, а у лиц с короткой паховой связкой минимальные (табл. 4).

Таким образом, топография поверхностной надчревной артерии, по сравнению с одноименной веной, вариабельна. Для уменьшения риска послеоперационных осложнений после реконструкции молочной железы «SIEA» лоскутом важно исследовать анатомию поверхностных надчревных сосудов еще на дооперационном этапе.

Таким образом, поверхностная надчревная артерия с обеих сторон наблюдалась в $70 \%$, а вена - в 100\% наблюдений. В 20\% данная артерия отмечалась лишь с одной стороны. В остальных наблюдениях поверхностная надчревная артерия отсутствовала с обеих сторон.

Относительно середины паховой связки поверхностная надчревная артерия всегда располагалась латерально $(0,8 \pm 0,2$ см), а вена медиально $(0,9 \pm 0,1$ см). Диаметр поверхностной надчревной артерии на уровне паховой связки

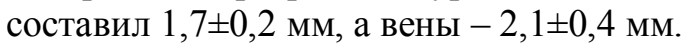

Расстояние между поверхностной надчревной артерией и веной на уровне паховой связки составило $1,6 \pm 0,5$ см. Установлена корреляционная зависимость между длиной паховой связки и расстоянием между сосудами на уровне паховой связки $(\mathrm{r}=0,75 ; \mathrm{p}=0,01)$.

Выявлены типы паховой связки: протяженная (длина более 15 см), обычная (12-15 см), короткая (менее $12 \mathrm{~cm}$ ). У лиц с протяженной паховой связкой наблюдались максимальные показатели расстояния между поверхностными надчревными сосудами, а у лиц с короткой паховой связкой минимальные.

\section{ЛИТЕРАТУРА}

1. Бежин А.И., Должиков А.А., Жуковский В.А., Нетяга А.А., Плотников Р.В. Экспериментальное обоснование применения новых поливинилиденфторидных эндопротезов с карбиновым покрытием для герниопластики // Вестник новых медицинских технологий. - 2007. - № 1. - С. 99-101.

2. Соколов Д.А., Квараихелия А.Г. Случай аномального строения локтевой артерии // Журнал анатомии и гистопатологии. - 2014. - № 3. - С. 71-72.

3. Судаков Д.В., Коровин Е.Н., Родионов О.В., Судаков О.В., Фурсова Е.A. Выбор тактики лечения осложнений сахарного диабета на основе нейросетевого моделирования // Системный анализ и управление в биомедицинских системах. - 2014. - Т. 13, № 3. - C. 592-597.

4. Черных А.В., Витчинкин В.Г., Якушева Н.В., Малеев Ю.В., Закурдаев Е.И., Болотских В.А., Спиuын В.B. Высокое отхождение лучевой и локтевой 
артерий // Журнал анатомии и гистопатологии. 2014. - T. 3, № 3. - С. 63-65.

5. Coroneos C.J., Heller A.M., Voineskos S.H., Avram R. SIEA versus DIEP Arterial Complications: A Cohort Study // Plast Reconstr Surg. - 2015. - Vol. 135, N 5. - P. 802-807.

6. Fathi M., Hatamipour E., Fathi H.R., Abbasi A. The anatomy of superficial inferior epigastric artery flap // Acta Cir Bras. - 2008. - Vol. 23, N 5. - P. 429-434.

7. Fukaya E., Kuwatsuru R., Iimura H., Ihara K., Sakurai $H$. Imaging of the superficial inferior epigastric vascular anatomy and preoperative planning for the SIEA flap using MDCTA // J Plast Reconstr Aesthet Surg. - 2011. - Vol. 64, N 1. - P. 63-68.

8. Kim B.J., Choi J.H., Kim T.H., Jin U.S., Minn K.W., Chang $H$. The superficial inferior epigastric artery flap and its relevant vascular anatomy in korean women // Arch Plast Surg. - 2014. - Vol. 41, N 6. P. 702-708.

9. Park J.E., Shenaq D.S., Silva A.K., Mhlaba J.M., Song D.H. Breast Reconstruction with SIEA Flaps: A Single-Institution Experience with 145 Free Flaps //
Plast Reconstr Surg. - 2016. - Vol. 137, N 6. P. 1682-1689.

10. Reardon C.M., O'Ceallaigh S., O'Sullivan S.T. An anatomical study of the superficial inferior epigastric vessels in humans // $\mathrm{Br}$ J Plast Surg. - 2004. Vol. 57, N 6. - P. 515-519.

11. Sarik J.R., Bank J., Wu L.C., Serletti J.M. Superficial Inferior Epigastric Artery: Learning Curve versus Reality // Plast Reconstr Surg. - 2016. - Vol. 137, N 1. - P. 1-6 - doi: 10.1097/PRS.0000000000001852.

12. Selber J.C., Samra F., Bristol M., Sonnad S.S., VegaS., Wu L., SerlettiJ.M. A head-to-head comparison between the muscle-sparing free TRAM and the SIEA flaps: is the rate of flap loss worth the gain in abdominal wall function? // Plast Reconstr Surg. - 2008. - Vol. 122, N 2. - P. 348-355. - doi: 10.1097/PRS.0b013e31817d60b0.

13. Wang X., Zhang K., Ma S., Meng Z. Applied anatomy of superficial inferior epigastric artery flap // Zhongguo Xiu Fu Chong Jian Wai Ke Za Zhi. - 2010. - Vol. 24, N 11. - P. 1357-1360. 